\title{
Newcomer status as a protective factor among Hispanic migrant workers for HIV risk
}

\author{
H. Virginia McCoy ${ }^{1}$, Nancy Shehadeh ${ }^{1}$, Muni Rubens ${ }^{1}{ }^{*}$ and Christi M. Navarro ${ }^{2}$ \\ ${ }^{\prime}$ Department of Health Promotion and Disease Prevention, Robert Stempel College of Public Health and Social Work, Florida International University, Miami, FL, USA \\ 2 Opportunities Industrialization Centers of South Florida, Fort Lauderdale, FL, USA
}

\section{Edited by:}

Badu Sarkodie, Ghana Health Service, Ghana

Reviewed by:

Marguerite Ro, Public Health - Seattle \& King County, USA

Karin Joann Opacich, University of

Illinois at Chicago, USA

${ }^{*}$ Correspondence:

Muni Rubens, Department of Health

Promotion and Disease Prevention, Robert Stempel College of Public

Health and Social Work, Florida International University, 11200 SW 8th Street, AHC // 559, Miami, FL 33199, USA

e-mail:mrube001@fiu.edu
The HIV rate among U.S. migrant workers is 10 times that of the national rate. The highly unstable lifestyle of migrant workers places them at heightened vulnerability to sexually transmitted infections; hence, there is a need to investigate the attitudes and sexual risk factors that may play a protective role in the transmission of HIV in this population. This study examines the association between attitudes and HIV risk behaviors among Hispanic male and female migrant workers $(n=255)$ and their length of stay (shorter length of stay as a protective factor) in Immokalee, FL, USA. Pearson's correlation and regression analyses were utilized to analyze the relationship between HIV risk behaviors (intention to use condoms and alcohol use) with length of stay in Immokalee. Longer length of stay positively correlated with number of drinks $(p<0.05)$ and frequency of drinks $(p<0.01)$ and negatively correlated with ethnic identity search $(p<0.05)$. Regression analysis showed that length of stay predicted both behavioral intention to use condoms $(p<0.05)$ and alcohol consumption $(p<0.05)$. The findings suggest that migrant workers who are new to Immokalee may have a higher likelihood of practicing protective HIV risk behaviors and having more favorable attitudes toward risk reduction than long-timers. This study might provide important new evidence on the drivers of multiple concurrent and potential protective factors against risky sexual behaviors among Hispanic migrant workers.

Keywords: migrant workers, length of stay, risky sexual behavior, behavioral intention, alcohol use

\section{INTRODUCTION}

One of the fastest growing, underserved populations in the US are migrant workers, presently exceeding over four million farmworkers (1). The majority of migrant workers are Hispanic and work in rural areas in the United States (2). This overlooked community carries a heavy burden of working in an area that involves high occupational risk as well as a number of other challenges that come along with working in the fields of the US and obstacles associated with migration, and in many cases are also undocumented (1-4). These challenges include the neighborhood they reside in, long hours, employment instability; stigma associated with migrant status, language barriers, and social isolation and place these individuals at heightened vulnerability in engaging in unhealthy coping behaviors, which increase their risk of sexually transmitted infections (STIs), especially HIV/AIDS (2). In addition to these many challenges, migrant workers have also been significantly affected by HIV/AIDS (5). The HIV seroprevalence rates reported for migrant workers are $2.6 \%$ to as high as $13.5 \%$, 10 times the national average (6-8).

For many migrant workers, especially Hispanics, social rules and norms appear to be less restrictive in the U.S. compared to their home countries (9). This perception, coupled with the stress that often accompanies working and functioning in a different culture, may encourage the use of high risk coping mechanisms such as heavy drinking and/or risky sexual behaviors $(3,10,11)$. Even when the norm involves consumption of large amounts of alcohol in their home countries, studies have shown that alcohol consumption increases with every year migrant workers reside in the U.S. $(9,12)$. Attitudes toward alcohol have also been found to reflect traditional gender roles (13). In a study among Latinomigrant workers in Beaufort, South Carolina, respondents indicated that drinking was not only widely accepted for men but encouraged as an act of proven masculinity and women who drank at all were not well accepted (9). Among Hispanics, males tend to consume higher levels of alcohol than their female counterparts (14). Traditional beliefs may contribute to high risk sexual behaviors among Hispanics (15). In Hispanic culture, sexual issues are highly personal and are not usually discussed, even with their sexual partners. In addition, beliefs in traditional gender roles lead to different standards for men and women. Such beliefs assume that men are more sexually active than women and that it is acceptable for men to have sexual relationships outside the purview of marriage. The gender role beliefs also tolerate sexual coercion (16).

Alcohol consumption, which is associated with uninhibited behaviors and impaired judgment, has been implicated as a risk factor for negative sexual outcomes, such as failure to use condoms and HIV transmission (17-19). Many studies have reported solicitation by sex workers, unprotected sex with casual partners, and participation in high risk sexual activities in the context of alcohol use $(13,17,18)$. Although many migrant workers engage in behaviors that increase their risk of HIV, there are several factors that serve to protect them from risk. For example, a study on Mexican migrants found that those who migrated to the U.S. at an older age were less likely to engage in substance abuse compared to those 
who migrated when they were younger. The study also demonstrated how the "healthy migrant" effect may not be exclusive to U.S. Hispanic populations, but may also be applied to migrant workers $(16,20)$.

Another study that investigated protective HIV behaviors of migrant workers was conducted in Durham, North Carolina, and Mexican-sending communities. This study illustrated that condom use during vaginal sex was higher among male migrants residing in the U.S. compared to non-migrants in Mexicansending communities, even though male migrants reported higher frequency of other HIV-related risk behaviors (21). Mexican migrants and non-migrants from their communities of origin differed significantly in their perception of the importance of condom use depending on how they perceived their sexual encounter as safe or unsafe. Mexican male migrants were more likely to believe in using condoms when engaging in sexual encounters and believed such preventative practices were of importance compared to their non-migrant counterparts. This acceptance of the use of condoms is claimed to be because of the higher incidence of multiple partners and commercial sex worker partners among these migrant workers (21-23).

Unfortunately, the literature on HIV/AIDS-risk behaviors among migrant workers is sparse, particularly in relation to protective factors. In addition to exploring sexual risk factors, it is equally important to investigate protective factors among migrant workers. This will aid researchers in designing interventions focused on encouraging the practice of protective behavior in HIV prevention strategies. Hence, the current study explores whether migrant workers' length of stay in Immokalee acts as a protective factor that influences their attitudes toward alcohol and sexual risk behaviors. Evidence from theoretical literature suggests that shorter length of stay could act as a protective factor influencing migrant workers' attitudes toward alcohol and sexual risk behaviors. We based our hypothesis that shorter length of stay could act as a protective factor on the health belief model. Perceived susceptibility is perceiving that oneself is at risk of sexually transmitted infection, including HIV (24-26). Increased perceived susceptibility to HIV infection has been related to increased protective behaviors like increased condom use $(24,27)$. We postulate that recent migrant workers have increased perceived susceptibility, an indicator of motivation to engage in protective sexual behaviors.

\section{MATERIALS AND METHODS DESIGN}

Participants from an ongoing two-group randomized study comparing the effectiveness of an enhanced/adapted cognitive behavioral program with a health promotion comparison program were used for this study. Participants in the larger study were migrant Hispanic and African American workers from Immokalee, a rural agricultural area in South Florida, who were recruited using targeted sampling. The aim of the parent study was to assess the effectiveness of two community-based interventions in reducing HIV risk behaviors and increasing health behaviors among alcohol and other drug using migrant workers (28).

The inclusion criteria for participants in this sub-study were being male or female Hispanics who spoke Spanish or English, and were age 18 years or older with a history of unprotected vaginal, anal, or oral sex and/or consumption of alcohol or other drugs in the previous 3 months. The interviews were executed in both languages, English and Spanish. These analyses were conducted among Hispanic migrant workers who participated in the baseline intake interviews $(n=255)$.

The migrant workers involved in the present study are a mix of migrant workers and seasonal workers within a 10 mile radius of Immokalee, Collier County, FL, USA. The study's definition of migrant workers conformed to the Public Health Services Act (1944) definition. The Public Health Services Act states that migrant workers are those individuals who are employed in agricultural labor, either seasonal or migratory, and live in temporary housing. The study criteria did not limit the definition of migrant workers to those who travel 75 miles or cross county lines to work. The study also included some people who work or worked indirectly in agriculture, such as in packing houses. However, the majority of migrant workers were farm workers. The Florida International University institutional review board examined (IRB) and approved the study protocol and research procedures.

\section{MEASURES \\ Length of stay}

Length of stay is the number of years the participants stayed at Immokalee, FL, USA.

\section{Alcohol consumption and frequency of drinking}

Alcohol use was measured by asking the participants how many standard drinks they had in the past month. Photographs of drinks and sample glass sizes were shown for verification of amount of alcohol intake. Frequency of drinking is the number of drinking days in the past month.

\section{Behavioral intentions}

Behavioral intentions to use condoms was measured using a 15item Behavioral Intentions Scale which focused on taking future actions to reduce the risk of transmitting HIV (e.g., "I will use a condom the next time I have sex"). This scale was derived by Klinkenberg (personal communication, 1998) by simplifying a measure used by Otto-Salaj et al. (29) and by adding an item about drinking (i.e., "I will use a condom the next time I have sex even if I've been drinking"). Items were scored on a 5-point scale ranging from 1 (definitely will not do) to 4 (definitely will do) and 77 (do not know). The responses were recoded with "Do not know" as mid-point. Cronbach's alpha for the Behavioral Intention Scale was 0.90 .

\section{Short inventory of problems}

A brief version of the short inventory of problems (SIP) was used for assessing negative consequences associated with the effects of alcohol and other drug use (e.g., "You have failed to do what is expected of you because of your drinking or drug use."). This measure is also known as the alcohol problems measure. This questionnaire is composed of a 9-item, modified version of the drinker inventory of consequences $(30,31)$. Items were scored on a Likert type scale with five responses for each item, from 0 (Never/Not at all) to 4 (Daily or almost daily/Very much), yielding a range of scores from 0 to 36 . The scale showed high reliability with a Cronbach's alpha of 0.93 . 


\section{Ethnic identity}

The multigroup ethnic identity measure (MEIM) was used to assess the ethnic identity level of the participants (32). MEIM is a 12-item questionnaire, which measures participants' level of comfort and attachment with their ethnic culture as well as members of their ethnic group. The MEIM focuses on analyzing areas of one's feelings of commitment and/or belonging to his/her ethnic group. The MEIM score also indicates the participant's comfort level with individuals from his or her own ethnic group $(32,33)$. The scores for each item use a Likert scale, ranging from 1 (strongly disagree) to 4 (strongly agree). A mean score was calculated to assess the level of ethnic identity search (first seven items) and commitment (last five items) of each participant. The higher the mean ethnic identity search score, the higher the developmental and cognitive component. The same was the case with ethnic identity commitment, the higher the commitment score, the higher the subject's emotional attachment to their ethnic group. The Cronbach's alpha of the ethnic identity search and commitment scales were 0.79 and 0.82 , respectively.

Table 1 shows the definitions and parameters of all variables used in the study.

\section{DATA ANALYSIS}

IBM SPSS Statistics for Windows, Version 21.0 was used to analyze the data (Armonk, NY, USA: IBM Corp.). Initially, descriptive statistics were done to describe the sample population. This was followed by Pearson's correlation of variables, which were used in the regression model to find the association between them. Finally, two separate multiple linear regression analyses were conducted after meeting all assumptions of linear regression. These analyses were conducted to predict behavioral intentions to use condoms and alcohol consumption rate. No evidence of mutlicollinearity was found among the predictor variables.

\section{RESULTS}

\section{PARTICIPANTS}

The data were collected at baseline, prior to participation in the intervention. The sample was composed of 255 Hispanic participants (Table 2). The mean age of the sample was around 38 years with a mean educational level of 7.25 years. Average length of stay of participants was around 14 years. This included people who were staying for less than a month to up to 55 years. Nearly, $68 \%$ of participants were seasonal migrant workers. The sample was primarily male $(n=201,78.8 \%)$ and majority were single $(n=195$, 76.5\%). Most of them spoke Spanish and were born in Mexico, Puerto Rico, and Cuba. Missing data was very minimal throughout the data set utilized for this study, not exceeding a range of $0-2 \%$. A mean replacement method was used to replace missing data.

\section{HIV RISK FACTORS}

The association between HIV risk behaviors and length of stay in this sample are illustrated in Table 3. The most frequent sexual risk behavior for this sample was having vaginal sex without a condom and multiple partners. Although there was a positive correlation between longer length of stay and number of unprotected vaginal sex episodes and number of partners, the association was not significant. However, there was a significant positive correlation between number of drinks $(r=0.31, p=0.028)$ and frequency of drinks $(r=0.28, p=0.006)$. In addition, length of stay was inversely associated with ethnic identity search $(r=-0.32$,

Table 1 | Variables used in the study.

\begin{tabular}{|c|c|c|}
\hline Variable name & Definition/measurement & Parameters \\
\hline Length of stay & $\begin{array}{l}\text { Number of years the participants stayed at Immokalee, FL, } \\
\text { USA. }\end{array}$ & Continuous variable \\
\hline Alcohol consumption & Number of standard drinks in the past month. & Continuous variable \\
\hline Frequency of drinking & Number of drinking days in the past month. & Continuous variable \\
\hline $\begin{array}{l}\text { Behavioral intentions scale to use } \\
\text { condoms (29) }\end{array}$ & $\begin{array}{l}\text { Individual's perceived or subjective intention of using } \\
\text { condom during sexual intercourse. }\end{array}$ & $\begin{array}{l}\text { Likert scale, ranging from } 1 \text { (definitely will not } \\
\text { do) to } 4 \text { (definitely will do) and } 77 \text { (do not know) }\end{array}$ \\
\hline $\begin{array}{l}\text { Short Inventory of problems (SIP) } \\
(30,31)\end{array}$ & $\begin{array}{l}\text { SIP measures physical, interpersonal, intrapersonal, social, } \\
\text { and impulsive consequences of alcohol use. }\end{array}$ & $\begin{array}{l}\text { Likert scale, ranging from } 0 \text { (never/not at all) to } 4 \\
\text { (daily or almost daily/very much) }\end{array}$ \\
\hline $\begin{array}{l}\text { Multigroup ethnic identity measure } \\
\text { (MEIM) (32) }\end{array}$ & $\begin{array}{l}\text { Individual's level of comfort and attachment with their ethnic } \\
\text { culture as well as members of their ethnic group. }\end{array}$ & $\begin{array}{l}\text { Likert scale, ranging from } 1 \text { (strongly disagree) } \\
\text { to } 4 \text { (strongly agree) }\end{array}$ \\
\hline Ethnic identity search (32) ${ }^{a}$ & Developmental and cognitive component of MEIM & $\begin{array}{l}\text { Likert scale, ranging from } 1 \text { (strongly disagree) } \\
\text { to } 4 \text { (strongly agree) }\end{array}$ \\
\hline Ethnic identity commitment (32) ${ }^{a}$ & Affective component of MEIM & $\begin{array}{l}\text { Likert scale, ranging from } 1 \text { (strongly disagree) } \\
\text { to } 4 \text { (strongly agree) }\end{array}$ \\
\hline $\begin{array}{l}\text { HIV risk factors: number of } \\
\text { unprotected vaginal sex episodes }\end{array}$ & $\begin{array}{l}\text { Number of unprotected vaginal sex episodes in the past } \\
\text { month. }\end{array}$ & Continuous variable \\
\hline HIV risk factors: number of partners & Number of sexual partners in the past month. & Continuous variable \\
\hline
\end{tabular}

${ }^{a}$ Ethnic identity search and ethnic identity commitment are sub-scales of MEIM. 
$p=0.007)$. Ethnic identity search was inversely correlated with number of unprotected vaginal sex episodes and number of partners. Behavioral intention to use condoms was negatively correlated with number of unprotected vaginal sex episodes $(r=-0.09$, $p=0.032$ ).

\section{PREDICTING HIV RISK}

The independent variables in the model predicting behavioral intentions to use condoms (Table 4) were demographic variables (age, gender, length of stay, marital status, and education), amount of alcohol consumed in last 30 days, ethnic identity subscales (ethnic identity commitment and ethnic identity explore) and SIP. The independent variables in the model predicting alcohol consumption (Table 5) were demographic variables (age, gender, length of stay, marital status, and education), ethnic identity commitment, ethnic identity explore, and SIP. In the model predicting behavioral intention, gender $(\beta=-0.021$, $p=0.032)$, length of stay $(\beta=-0.024, p=0.042)$, ethnic identity search $(\beta=0.023, p=0.007)$, and $\operatorname{SIP}(\beta=0.137, p=0.012)$

Table 2 | Demographic characteristics of the sample $(n=255)$.

\begin{tabular}{lc}
\hline Variables & Values \\
\hline Age $(M \pm S D)$ & $38.46 \pm 11.59$ \\
Education $(M \pm S D)$ & $7.25 \pm 3.35$ \\
Gender $n(\%)$ & \\
Male & $201(78.8 \%)$ \\
Female & $54(21.2 \%)$ \\
Marital status $n(\%)$ & \\
Single & $195(76.5 \%)$ \\
Married & $60(23.5 \%)$ \\
Language $n(\%)$ & \\
$\quad$ English & $52(20.4 \%)$ \\
Spanish & $202(79.2 \%)$ \\
Country of birth $n(\%)$ & $154(60.4 \%)$ \\
USA & $101(39.6 \%)$ \\
Others &
\end{tabular}

significantly predicted behavioral intention to use condoms. In the second regression model, gender $(\beta=-32.928, p=0.026)$, length of stay $(\beta=-25.856, p=0.035)$, and $\operatorname{SIP}(\beta=3.575, p=0.016)$ significantly predicted alcohol consumed in last 30 days.

\section{DISCUSSION}

This study was focused on exploring whether length of stay is protective in HIV risk behaviors. Several important findings in this Hispanic migrant worker study merit discussion. Hispanic migrant workers who lived in Immokalee for less time demonstrated higher intentions to use condoms and consumed less alcohol than their long-timer peers which may be a reflection of how they perceived their environment.

Condom use was inversely associated with length of stay, indicating that condom use was common among the more recent migrant workers or newcomers. Condom use was also more common in males when the full sample was examined. This finding is consistent with a previous study which revealed that newcomers were more likely to use condoms and get screened for HIV than long-timers (22). The number of sexual partners was also inversely correlated with length of stay. The relationship of length of stay and condom use and number of sexual partners might be due to their perception of casual sexual encounters in the U.S. as being risky. This study illustrates that more recent migrants tend to perceive their new environment as more risky and acknowledge that the casual sexual episodes which they pursue are risky as well. For instance, a study by Organista and Ehrlich (34) reported that migrant workers who had partners back in Mexico were about four times more likely to use condoms with casual female sexual partners than males who have their wives or partners accompanying them. Condom use variation by gender is in line with findings reported by several other study results that male migrants were more likely to believe in using condoms when engaging in sexual encounters and believed such preventative practices were of importance compared to their non-migrant counterparts $(12,21)$.

Predictors of the number of drinks in the last 30 days were gender, length of stay, and alcohol problems measure (sexual inventory of problems). Males were more likely to consume more alcohol

Table 3 | Correlations among variables in the regression model $(\boldsymbol{n}=\mathbf{2 5 5})$.

\begin{tabular}{|c|c|c|c|c|c|c|c|c|c|}
\hline Variables & 1 & 2 & 3 & 4 & 5 & 6 & 7 & 8 & 9 \\
\hline Length of stay & - & - & - & - & - & - & - & - & - \\
\hline Number of unprotected vaginal sex episodes & 0.31 & - & - & - & - & - & - & - & - \\
\hline Number of partners & 0.38 & 0.14 & - & - & - & - & - & - & - \\
\hline Number of drinks & $0.31^{*}$ & $0.06^{*}$ & 0.13 & - & - & - & - & - & - \\
\hline Frequency of drinking & $0.28^{* *}$ & 0.21 & 0.15 & 0.25 & - & - & - & - & - \\
\hline SIP & -0.21 & 0.11 & 0.09 & 0.09 & $0.14^{*}$ & - & - & - & - \\
\hline Behavioral intention & -0.08 & $-0.09^{*}$ & -0.30 & 0.12 & 0.24 & 0.07 & - & - & - \\
\hline El search & $-0.32^{*}$ & $-0.20^{*}$ & $-0.21^{*}$ & 0.18 & 0.20 & 0.14 & 0.24 & - & - \\
\hline El commitment & -0.17 & $0.31 *$ & 0.02 & 0.06 & 0.08 & 0.17 & 0.12 & 0.28 & - \\
\hline
\end{tabular}

${ }^{*} p<0.05,{ }^{*} p<0.01$.

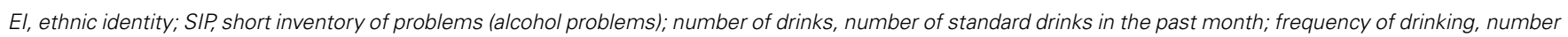
of drinking days in the past month; behavioral intention, behavioral intention to use condoms.

All Sexual acts and consumption of alcohol refer to the last 30 days. 
Table 4 | Multiple linear regression predicting behavioral intentions to use condoms $(n=255)$.

\begin{tabular}{lccc}
\hline Independent variables & Beta estimate & Standard error & $\boldsymbol{p}$-value \\
\hline Age & 0.014 & 0.003 & 0.678 \\
Gender* $^{*}$ & -0.020 & 0.014 & 0.031 \\
Length of stay* $^{*}$ & -0.023 & 0.014 & 0.041 \\
Marital status & -0.017 & 0.007 & 0.318 \\
Education & 0.015 & 0.013 & 0.628 \\
Alcohol consumption $^{\mathrm{a}}$ & 3.926 & 0.009 & 0.355 \\
Number of partners $^{\text {El search**a }}$ & -1.025 & 0.016 & 0.059 \\
El commitment $^{\mathrm{a}}$ & 0.026 & 0.016 & 0.007 \\
SIP* $^{*}$ & -0.031 & 0.021 & 0.269 \\
& 0.133 & 0.008 & 0.012
\end{tabular}

${ }^{*} p<0.05,{ }^{*} p<0.01$.

aTransformed value.

El, ethnic identity; SIP, short inventory of problems; alcohol consumption, number of standard drinks in the past month.

All Sexual acts and consumption of alcohol refer to the last 30 days.

Table 5 | Multiple linear regression predicting alcohol consumption ${ }^{\text {a }}$ $(n=255)$.

\begin{tabular}{lrrr}
\hline Independent variables & Beta estimate & Standard error & $\boldsymbol{p}$-value \\
\hline Age & -0.263 & 0.272 & 0.529 \\
Gender* $^{*}$ & -32.928 & 12.846 & 0.026 \\
Length of stay* $_{\text {Marital status }}$ & 25.856 & 8.456 & 0.035 \\
Education $^{*}$ & 9.464 & 5.367 & 0.735 \\
El search $^{a}$ & -2.464 & 1.935 & 0.332 \\
El commitment $^{a}$ & -12.648 & 9.354 & 0.246 \\
SIP* $^{*}$ & 14.464 & 8.457 & 0.273 \\
\hline
\end{tabular}

${ }^{*} p<0.05$.

${ }^{a}$ Transformed value.

El, ethnic identity; SIP, short inventory of problems.

All sexual acts and consumption of alcohol refer to the last 30 days.

than their female counterparts. Gender variation in alcohol use was reported by other studies as well $(14,35,36)$. Length of stay was a significant predictor of alcohol consumption. Shorter length of stay was directly associated with fewer drinking episodes, as well as fewer drinks in the last 30 days. Previous studies reported newcomer frequency of drinking episodes to be less than migrant workers who have been in the U.S. longer (13). Although actual frequency of drinking episodes was not very high, the number of drinks per episode was, suggesting heavy or binge drinking. Alcohol consumption has been reported to increase with every year a migrant worker is in the U.S., which was evident in this study. Studies suggest that migrant workers who are not recent arrivals to the U.S. tend to be at high risk for alcohol abuse and have been found to consume high levels of alcohol to cope with their stress of working and functioning in a different culture $(13,37)$.

The alcohol problems measure was a significant predictor for both alcohol consumption and intentions to use condoms.
Higher alcohol problems were associated with higher alcohol consumption as well as lower intentions to use condoms. Previous studies have shown negative sexual outcomes under the influence of alcohol among migrant workers $(18,34)$. Alcohol consumption, in many cases, may lead to risky sexual behaviors, ultimately placing the participant at higher risk for HIV. While our findings correspond with past studies on sexual risk behaviors and alcohol use, this study makes significant contributions to the existing literature on the protective role of length of stay of migrant workers in the U.S. Our findings assert the association of newcomer status with high number of condom use and lesser alcohol intake. We further add to the existing literature the important finding that length of stay could predict intention to use condom and alcohol consumption. This finding might be due to the unique perception of newcomers about the new environmental and vulnerability factors to which they are exposed.

Ethnic identity was one of the contributing factors that influenced intentions to use condoms. Previous research has demonstrated that individuals with a stronger connection with their ethnic group (ethnic identity) served as a protective factor with risky sexual behaviors and risky sexual attitudes and beliefs of individuals $(38,39)$. This study demonstrated that those migrant workers who were more interested in learning about their ethnic origin also practiced protective HIV risk behavior and had a more positive attitude to engaging in such protective behaviors.

Studies have shown that, although traditional beliefs may lead to high risk sexual behaviors among Hispanics, some of these beliefs may change with time due to acculturation (40). This is contrary to the findings of this study. This could be due to the fact that more than half of the participants were born in the U.S. However, more studies are needed to explore how migrant workers accommodate both mainstream and heritage culture.

There are some limitations to this study. Since this is a report on the cross-sectional portion of the study, it limits the ability to draw conclusions or make predictions of causal relationships. The sample size was also relatively small. The data reported is self-reported, which may increase recall bias. Finally, previous studies have measured drinking by number of drinks per drinking episode, which was not available in this study. This lack of information may limit the ability to draw comparisons with previous studies.

\section{CONCLUSION}

Notwithstanding the methodological limitations, the findings of this study translate into relevant conclusions that newcomer status may be a protective factor against HIV risk behaviors, including condom use and alcohol consumption. As one of the few studies to date to explore shorter length of stay as a protective factor, this research provides important new evidence on the drivers of multiple concurrent and potential protective factors. These findings have important implications for health professionals who work to prevent substance abuse, sexual risk, and other HIV risk behaviors among migrant workers.

This study indicates that risk prevention interventions targeting condom use and alcohol use should be focused more on longtimers to provide them with the skills to support and enhance levels of positive change. A successful approach should meet the needs of individual migrant worker by assessing differences in 
problem behaviors by gender. Males, especially, need to be targeted in future HIV prevention programs since previous studies, as well as this study suggest that Hispanic males tend to be at highest risk for alcohol abuse. Future research should take into account the length of time migrant workers are in the community and include comparisons of newcomers and those who have been in the community for a longer period of time to better understand the effect of migration on HIV risk behaviors. In addition, investigations should explore if and how behavior of newcomers influences protective performance, or if unobserved factors might be implicated in this phenomenon.

\section{AUTHOR CONTRIBUTIONS}

H. Virginia McCoy and Nancy Shehadeh designed and conceptualized the study. Nancy Shehadeh and Muni Rubens collected the data and performed data analyses. H. Virginia McCoy, Nancy Shehadeh, Muni Rubens, and Christi M. Navarro wrote portions of the manuscript.

\section{ACKNOWLEDGMENTS}

The funding for this study was from Grant Number: 5R01AA 015810, National Institute on Alcohol and Alcohol Abuse. We would like to extend our thanks to all the men and women who travel across this great land to work in agriculture, construction, and other industries and would like to dedicate this intervention to those hard-working individuals. The authors would like to express heart-felt appreciation to Lucy Maharaj, Emma Ergon, and the Immokalee partner staff, colleagues, students, clinicians and other researchers who have been a source of support in the development of the P.E.E.R./H.E.A.L.T.H.Y. interventions.

\section{REFERENCES}

1. Persichino J, Ibarra L. HIV and Latino migrant workers in the USA. Ethn Racial Stud (2012) 35(1):120-34. doi:10.1080/01419870.2011.594167

2. Mann L, Valera E, Hightow-Weidman LB, Barrington C. Migration and HIV risk: life histories of Mexican-born men living with HIV in North Carolina. Cult Health Sex (2014) 16:1-15. doi:10.1080/13691058.2014.918282

3. Kissinger P, Althoff M, Burton N, Schmidt N, Hembling J, Salinas O, et al. Prevalence, patterns and predictors of substance use among Latino migrant men in a new receiving community. Drug Alcohol Depend (2013) 133(3):814-24. doi:10.1016/j.drugalcdep.2013.08.031

4. Organista KC, Worby PA, Quesada J, Arreola SG, Kral AH, Khoury S. Sexual health of Latino migrant day labourers under conditions of structural vulnerability. Cult Health Sex (2013) 15(1):58-72. doi:10.1080/13691058.2012.740075

5. Coppel J, Dumont J-C, Visco I. Trends in Immigration and Economic Consequences. Paris: OECD (2001).

6. Fitzgerald K, Chakraborty J, Shah T, Khuder S, Duggan J. HIV/AIDS knowledge among female migrant farm workers in the Midwest. J Immigr Health (2003) 5(1):29-36. doi:10.1023/A:1021000228911

7. Hansen E, Donohoe M. Health issues of migrant and seasonal farmworkers. J Health Care Poor Underserved (2003) 14(2):153-64. doi:10.1177/ 1049208903014002001

8. Sanchez J, Silva-Suarez G, Serna CA, De La Rosa M. The Latino migrant worker HIV prevention program: building a community partnership through a community health worker training program. Fam Community Health (2012) 35(2):139-46. doi:10.1097/FCH.0b013e3182465153

9. Apostolopoulos Y, Sonmez S, Kronenfeld J, Castillo E, McLendon L, Smith D. STI/HIV risks for Mexican migrant laborers: exploratory ethnographies. J Immigrant Minor Health (2006) 8(3):291-2. doi:10.1007/s10903-006-9334-2

10. Deren S, Shedlin M, Decena CU, Mino M. Research challenges to the study of HIV/AIDS among migrant and immigrant Hispanic populations in the United States. J Urban Health (2005) 82:iii13-25. doi:10.1093/jurban/jti060
11. Kissinger P, Kovacs S, Anderson-Smits C, Schmidt N, Salinas O, Hembling J, et al. Patterns and predictors of HIV/STI risk among Latino migrant men in a new receiving community. AIDS Behav (2012) 16(1):199-213. doi:10.1007/s10461011-9945-7

12. Duke MR, Gómez Carpinteiro FJ. The effects of problem drinking and sexual risk among Mexican migrant workers on their community of origin. Hum Organ (2009) 68(3):328-39.

13. Winkleby MA, Snider J, Davis B, Jennings MG, Ahn DK. Cancer-related health behaviors and screening practices among Latinos: findings from a community and agricultural labor camp survey. Ethn Dis (2003) 13(3):376-86.

14. Villarejo D, McCurdy SA, Bade B, Samuels S, Lighthall D, Williams D. The health of California's immigrant hired farmworkers. Am J Ind Med (2010) 53(4):387-97. doi:10.1002/ajim.20796

15. Marín BV. HIV prevention in the Hispanic community: sex, culture, and empowerment. J Transcult Nurs (2003) 14(3):186-92. doi:10.1177/ 1043659603014003005

16. Marín BV, Gómez CA, Tschann JM, Gregorich SE. Condom use in unmarried Latino men: a test of cultural constructs. Health Psychol (1997) 16(5):458. doi:10.1037/0278-6133.16.5.458

17. Organista KC, Kubo A. Pilot survey of HIV risk and contextual problems and issues in Mexican/Latino migrant day laborers. J Immigrant Minor Health (2005) 7(4):269-81. doi:10.1007/s10903-005-5124-0

18. Shuper PA, Neuman M, Kanteres F, Baliunas D, Joharchi N, Rehm J. Causal considerations on alcohol and HIV/AIDS - a systematic review. Alcohol Alcohol (2010) 45(2):159-66. doi:10.1093/alcalc/agp091

19. Malow RM, Dévieux JG, Stein JA, Rosenberg R, Lerner BG, Attonito J, et al. Neurological function, information-motivation-behavioral skills factors, and risk behaviors among HIV-positive alcohol users. AIDS Behav (2012) 16(8):2297-308. doi:10.1007/s10461-012-0246-6

20. Ulibarri MD, Raj A, Amaro H. Love, sex, and power revisited. The integration of a gendered context. In: Organista KC, editor. HIV Prevention with Latinos: Theory, Research, and Practice. New York: Oxford University Press (2012). p. 63-82. doi:10.1093/acprof:oso/9780199764303.003.0004

21. Prado G, Pantin H. Reducing substance use and HIV health disparities among Hispanic youth in the USA: the Familias Unidas program of research. Intervenc Psicosoc (2011) 20(1):63. doi:10.5093/in2011v20n1a6

22. Magis-Rodriguez C, Lemp G, Hernandez M, Sanchez M, Estrada F, Bravo-Garcia E. Going north: Mexican migrants and their vulnerability to HIV. J Acquir Immune Defic Syndr (2009) 51:S21-5. doi:10.1097/QAI.0b013e3181a26433

23. Ojeda VD, Strathdee SA, Lozada R, Rusch ML, Fraga M, Orozovich P, et al. Associations between migrant status and sexually transmitted infections among female sex workers in Tijuana, Mexico. Sex Transm Infect (2009) 85(6):420-6. doi:10.1136/sti.2008.032979

24. Sheeran P, Abraham C, Orbell S. Psychosocial correlates of heterosexual condom use: a meta-analysis. Psychol Bull (1999) 125(1):90. doi:10.1037/0033-2909. 125.1 .90

25. Kalichman S, Malow R, Dévieux J, Stein JA, Piedman F. HIV risk reduction for substance using seriously mentally ill adults: test of the information-motivationbehavior skills (IMB) model. Community Ment Health J (2005) 41(3):277-90. doi:10.1007/s10597-005-5002-1

26. Fisher JD, Fisher WA. Changing AIDS-risk behavior. Psychol Bull (1992) 111(3):455. doi:10.1037/0033-2909.111.3.455

27. Ashery RS, Wild J, Zhao Z, Rosenshine N, Young P. The Wheel Project: women helping to empower and enhance lives. J Subst Abuse Treat (1997) 14(2):113-21. doi:10.1016/S0740-5472(96)00129-8

28. McCoy HV, Hlaing WM, Ergon-Rowe E, Samuels D, Malow R. Lessons from the fields: a migrant HIV prevention project. Public Health Rep (2009) 124(6):790.

29. Otto-Salaj LL, Heckman TG, Stevenson LY, Kelly JA. Patterns, predictors and gender differences in HIV risk among severely mentally ill men and women. Community Ment Health J (1998) 34(2):175-90. doi:10.1023/A:1018745119578

30. Bowen S, Witkiewitz K, Dillworth TM, Chawla N, Simpson TL, Ostafin BD, et al. Mindfulness meditation and substance use in an incarcerated population. Psychol Addict Behav (2006) 20(3):343. doi:10.1037/0893-164X.20.3.343

31. Miller WR, Tonigan JS, Longabaugh R. The Drinker Inventory of Consequences (DrInC): An Instrument for Assessing Adverse Consequences of Alcohol Abuse: Test Manual. Rockville, Maryland: US Department of Health and Human Services, Public Health Service, National Institutes of Health, National Institute on Alcohol Abuse and Alcoholism (1995). 
32. Phinney JS. The multigroup ethnic identity measure a new scale for use with diverse groups. J Adolesc Res (1992) 7(2):156-76. doi:10.1177/ 074355489272003

33. Phinney JS, Cantu CL, Kurtz DA. Ethnic and American identity as predictors of self-esteem among African American, Latino, and White adolescents. J Youth Adolesc (1997) 26(2):165-85. doi:10.1023/A:1024500514834

34. Organista KC, Ehrlich SF. Predictors of condom use in Latino migrant day laborers. Hisp J Behav Sci (2008) 30(3):379-96. doi:10.1177/0739986308320881

35. Kim-Godwin YS, Fox JA. Gender differences in intimate partner violence and alcohol use among Latino-migrant and seasonal farmworkers in rural southeastern North Carolina. J Community Health Nurs (2009) 26(3):131-42. doi:10.1080/07370010903034474

36. Zamboanga BL, Raffaelli M, Horton NJ. Acculturation status and heavy alcohol use among Mexican American college students: investigating the moderating role of gender. Addict Behav (2006) 31(12):2188-98. doi:10.1016/j.addbeh.2006. 02.018

37. Worby PA, Organista KC. Alcohol use and problem drinking among male Mexican and Central American Im/migrant laborers a review of the literature. Hisp J Behav Sci (2007) 29(4):413-55. doi:10.1177/0739986307308765

38. Espinosa-Hernández G, Lefkowitz ES. Sexual behaviors and attitudes and ethnic identity during college. J Sex Res (2009) 46(5):471-82. doi:10.1080/ 00224490902829616

39. Nyembezi A, Resnicow K, Ruiter RA, van den Borne B, Sifunda S, Funani I, et al. The association between ethnic identity and condom use among young men in the Eastern Cape Province, South Africa. Arch Sex Behav (2014) 43:1-7. doi:10.1007/s10508-014-0307-1

40. Rojas-Guyler L, Ellis N, Sanders S. Acculturation, health protective sexual communication, and HIV/AIDS risk behavior among Hispanic women in a large Midwestern city. Health Educ Behav (2005) 32(6):767-79. doi:10.1177/ 1090198105277330

Conflict of Interest Statement: The authors declare that the research was conducted in the absence of any commercial or financial relationships that could be construed as a potential conflict of interest.

Received: 06 May 2014; accepted: 15 October 2014; published online: 07 November 2014.

Citation: McCoy HV, Shehadeh N, Rubens M and Navarro CM (2014) Newcomer status as a protective factor among Hispanic migrant workers for HIV risk. Front. Public Health 2:216. doi: 10.3389/fpubh.2014.00216

This article was submitted to Public Health Education and Promotion, a section of the journal Frontiers in Public Health.

Copyright (C) 2014 McCoy, Shehadeh, Rubens and Navarro. This is an open-access article distributed under the terms of the Creative Commons Attribution License (CC $B Y)$. The use, distribution or reproduction in other forums is permitted, provided the original author(s) or licensor are credited and that the original publication in this journal is cited, in accordance with accepted academic practice. No use, distribution or reproduction is permitted which does not comply with these terms. 